\title{
ESTUDO DAS ISOTERMAS DE EQUILÍBRIO DO FARELO DE SOJA ${ }^{1}$
}

\author{
Gianini R. LUZ², Luiza H.C.D. SOUSA², Luiz M.M. JORGE², Paulo R. PARAÍSO ${ }^{2, *}$
}

\section{RESUMO}

Este trabalho tem como objetivo estudar o comportamento das isotermas de equilíbrio do farelo de soja. Para tanto, foram realizados experimentos utilizando-se o método estático com soluções salinas saturadas a 50,60 e $70^{\circ} \mathrm{C}$. Os dados obtidos foram ajustados às equações existentes na literatura, utilizando-se o software Statistica $6.0^{\circledast}$. Os resultados indicam que a temperatura não exerce influência significativa sobre os valores da umidade de equilíbrio do farelo de soja na faixa de condições experimentais exploradas e que os modelos de HALSEY e de LUIKOV podem ser utilizados para prever a umidade de equilíbrio do farelo soja. Palavras-chave: farelo de soja, umidade de equilíbrio, isoterma, secagem.

\section{SUMMARY}

STUDY OF THE EQUILIBRIUM ISOTHERMS OF SOYBEAN MEAL. This work has as aim to study the behavior of the isotherms of the soybean meal. Therefore, experiments had been carried out by using the static method with saturated salt solution at 50,60 and $70^{\circ} \mathrm{C}$. The data had been adjusted to the equations of the literature, using Statistica software $6.0^{\circledast}$. The results indicated that the temperature does not affect significantly the moisture equilibrium values of the soybean meal in the range of temperatures studied and that the LUIKOV and the HALSEY models can be used to foresee the moisture equilibrium of the soybean meal.

Keywords: soybean meal, moisture of the equilibrium, isotherms, drying.

\section{1 - INTRODUÇÃO}

O farelo de soja, resultante da extração do óleo de soja, possui grande importância no mercado agropecuário brasileiro e mundial como ração animal e complemento alimentar humano, conforme salientado por TEIXEIRA [12].

O farelo de soja, conforme PARAÍSO [7], representa $70 \%$ da massa sólida do grão de soja, sendo que $51 \%$ correspondem às proteínas, $43 \%$ aos carboidratos e $6 \%$ às cinzas.

A soja passa por várias fases até a obtenção do farelo de soja descritas por SARTORI [10] e PARAÍSO [7], que são: silagem, limpeza, secagem dos grãos, quebra dos grãos, cozimento, laminação, expansão, extração, dessolventização-tostagem, secagem do farelo de soja, peletização, moagem e expedição.

A secagem do farelo de soja é uma etapa importante deste processo, pois aumenta o tempo de validade do farelo e reduz custos de transporte. Esta operação, normalmente apresenta elevado consumo de energia, que pode ser de até $80 \mathrm{~kg}$ de vapor por tonelada de soja processada, aumentando os custos operacionais e prejudicando o meio ambiente em termos de aquecimento e emissões poluentes, segundo JONGENEELEN [5]. Sendo assim,

\footnotetext{
${ }^{1}$ Recebido para publicação em 19/7/2005. Aceito para publicação em 28/4/2006 (001577)

${ }^{1}$ Universidade Estadual de Maringá/DEQ, Avenida Colombo, 5790 - CEP 87020-900 - Maringá - Paraná - Brasil - (44) 3261-4752

E-mail:paulo@deq.uem.br

${ }^{2} \mathrm{DEQ} / U E M$, e-mail: gianiniluz@yahoo.com.br

${ }^{3} \mathrm{DEQ} / \mathrm{UEM}$,e-mail:lmmj@deq.uem.br

${ }^{4} \mathrm{DEQ} / \mathrm{UEM}$,luizah@deq.uem.br

*A quem a correspondência deve ser enviada
}

na otimização do processo de secagem do farelo de soja, torna-se desejável estudar o processo, a fim de reduzir o consumo de energia e os danos ao meio ambiente. Para tanto, normalmente desenvolve-se um modelo matemático do processo de secagem, cuja simulação e otimização realistas dependem do conhecimento de dados de equilíbrio confiáveis do farelo de soja.

A umidade de equilíbrio é a umidade que o produto irá apresentar a uma determinada umidade relativa do ar e em uma determinada pressão e temperatura. Os dados de umidade de equilíbrio variam com o tipo de sólido e podem ser determinados, experimentalmente, por métodos estáticos ou dinâmicos, conforme BARROZO [1].

Estes dados de umidade de equilíbrio raramente são encontrados na literatura, uma destas exceções é o trabalho de PIXTON e WARBURTON [9], que apresentaram dados para farelo de soja a 15,25 e $35^{\circ} \mathrm{C}$, uma faixa de temperatura adequada para o armazenamento. Podendo notar-se, a partir daí, a necessidade do conhecimento das umidades de equilíbrio para o farelo a temperaturas superiores a $35^{\circ} \mathrm{C}$ com a finalidade de aplicação em processos industriais. Esses dados experimentais podem ser ajustados usando equações da literatura adequadas para cereais.

As correlações encontradas na literatura, normalmente, são empíricas e semi-empíricas, uma vez que nenhum modelo teórico foi capaz de predizer com precisão o teor de umidade de equilíbrio em todas as faixas de temperatura, conforme apresentado por BROOKER et al. [2].

As equações utilizadas para o ajuste neste trabalho foram as de LUIKOV (1978), de HALSEY (1976) e de HENDERSON-THOMPSON (1968), obtidas em SOUSA et al. [11] para estimar umidade de equilíbrio e podem ser vistas na Tabela 1 . 
TABELA 1 - Equações para o cálculo da umidade de equilíbrio $(\mathrm{Xe})$

\begin{tabular}{ll}
\hline Correlação de LUIKOV (1978) & $\mathrm{Xe}=\frac{\mathrm{A}_{1}}{\left(1+\mathrm{A}_{2} \mathrm{~T} \ln \left(\frac{1}{\mathrm{UR}}\right)\right)}$ \\
Correlação de HALSEY (1976) & $\mathrm{Xe}=\mathrm{A}_{1}\left(\mathrm{~T} \operatorname{In}\left(\frac{1}{\mathrm{UR}}\right)\right)^{-\mathrm{A}_{2}}$ \\
$\begin{array}{l}\text { Correlação de } \\
\text { HENDERSON-THOMPSON (1968) }\end{array}$ & $\mathrm{Xe}=\left(\frac{-\ln (1-\mathrm{UR})}{\mathrm{A}_{1} T+\mathrm{A}_{2}}\right)$ \\
\hline
\end{tabular}

Neste contexto, o objetivo deste trabalho foi determinar, experimentalmente, os dados de umidade de equilíbrio do farelo de soja, utilizando soluções salinas saturadas a 50, 60 e $70^{\circ} \mathrm{C}$, segundo a metodologia descrita por BARROZO [1], MUJUNDAR [6] e GREENSPAN [4], a qual é baseada em um procedimento estático. Posteriormente, os cálculos foram realizados no software Statistica $6.0^{\circledR}$ através do método de iteração de Rosenbrock e Quasi-Newton.

\section{2 - MATERIAL E MÉTODOS}

\section{1 - Materiais}

A dessorção do farelo foi realizada com amostras de $0,15 \mathrm{~cm}$ de tamanho médio, retiradas na saída do equipamento denominado de Dessolventizador/Tostador, fornecidas pela Cocamar (Cooperativa Agrícola de Maringá), localizada na Região Noroeste do Paraná, empregando-se soluções salinas de $\mathrm{LiCl}, \mathrm{CH}_{3} \mathrm{CO}_{2} \mathrm{~K}$, $\mathrm{K}_{2} \mathrm{CO}_{3}, \mathrm{NaNO}_{2}$, NaCl e $\mathrm{KCl}$ que foram armazenadas juntamente com a amostra em vidros cilíndricos. Os recipientes apresentavam diâmetro de $7,6 \mathrm{~cm}$, altura de $12 \mathrm{~cm}$ e tela localizada dentro deste com a altura 6 $\mathrm{cm}$ em relação ao fundo do vidro e sobre a solução para sustentar o farelo durante o processo de desidratação, conforme a Figura 1 .

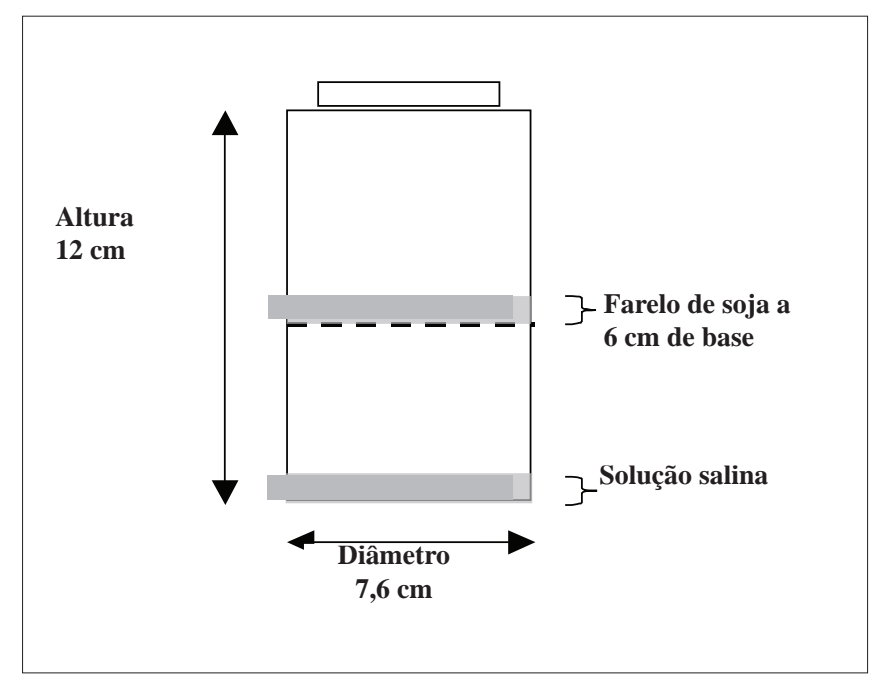

FIGURA 1 - Esquema para medição de umidade estática

\section{2 - Metodologia experimental}

A desidratação das amostras de farelo de soja foi realizada nos laboratórios da UEM, segundo o procedimento descrito abaixo. Cada solução salina foi colocada cuidadosamente no fundo dos recipientes e as amostras de $0,025 \mathrm{~kg}$ ficaram dispostas sobre uma tela. Em seguida, fecharam-se os recipientes hermeticamente.

Posteriormente, as amostras foram colocadas em estufa a $50^{\circ} \mathrm{C}$ e pesadas até atingir peso constante em três medidas sucessivas. Considerou-se que, normalmente este processo leva em torno de 21 dias para ser concluído. Na etapa seguinte, coloca-se a amostra, de forma isolada, para secar na estufa sob temperatura fixa de $105 \pm 2^{\circ} \mathrm{C}$, e então, é pesada após $24 \mathrm{~h}$ ou até atingir peso constante (o que indica que a secagem foi concluída). Também é medida a umidade relativa, UR, e a temperatura do ar, $T$. O processo foi repetido para as temperaturas de 60 e $70^{\circ} \mathrm{C}$. Todas as medidas foram realizadas em triplicata para dar confiabilidade aos dados obtidos. Os sais foram escolhidos a partir dos dados apresentados por MUJUNDAR [6].

\section{3 - Metodologia de ajuste das isotermas de equilíbrio}

Com os dados da umidade de equilíbrio, $\mathrm{Xe}$, da umidade relativa do ar, UR, e as equações de LUIKOV, HALSEY e HENDERSON-THOMPSON, ajustaram-se os parâmetros de cada uma dessas equações pela técnica da regressão não-linear, utilizando o software Statistica $6.0^{\circledR}$.

Posteriormente, conhecendo-se os parâmetros ajustados, $A_{n}$, construíram-se as isotermas de equilíbrio e obtiveramse os coeficientes de correlação, $R^{2}$, e o somatório dos desvios quadráticos, $\phi$, sendo $\phi=\Sigma\left(\left.X e\right|_{\text {modelo }}-\left.X e\right|_{\text {experimental }}\right)^{2}$, a fim de verificar qual delas melhor representa os dados experimentais.

\section{3 - RESULTADOS E DISCUSSÃO}

Na Tabela 2, são apresentados os valores médios experimentais de umidade de equilíbrio, Xe, e umidade relativa, UR, para cada solução salina nas temperaturas 50,60 e $70^{\circ} \mathrm{C}$. As concentrações das soluções salinas foram especificadas conforme PERRY e CHILTON [8], a fim de obterem-se os valores de UR apresentados na Tabela 2.

TABELA 2 - Dados experimentais de umidade de equilíbrio, $X_{e}$, em função da umidade relativa, $U R$

\begin{tabular}{lcccccc}
\hline \multirow{2}{*}{ Solução salina } & \multicolumn{2}{c}{$\mathbf{5 0 ^ { \circ } \mathbf { C }}$} & \multicolumn{2}{c}{$\mathbf{6 0 ^ { \circ } \mathbf { C }}$} & \multicolumn{2}{c}{$\mathbf{7 0}^{\circ} \mathbf{C}$} \\
\cline { 2 - 7 } & UR & $\mathbf{X}_{\mathrm{e}}$ & UR & $\mathbf{X}_{\mathrm{e}}$ & UR & $\mathbf{X}_{\mathrm{e}}$ \\
\hline $\mathrm{LiCl}$ & 0,111 & 0,034 & 0,110 & 0,029 & 0,109 & 0,023 \\
$\mathrm{CH}_{3} \mathrm{CO}_{2} \mathrm{~K}$ & 0,192 & 0,046 & 0,179 & 0,035 & 0,166 & 0,033 \\
$\mathrm{~K}_{2} \mathrm{CO}_{3}$ & 0,433 & 0,068 & 0,433 & 0,078 & 0,433 & 0,141 \\
$\mathrm{NaNO}_{2}$ & 0,594 & 0,106 & 0,574 & 0,101 & 0,554 & 0,093 \\
$\mathrm{NaCl}$ & 0,744 & 0,185 & 0,741 & 0,172 & 0,738 & 0,184 \\
$\mathrm{KCl}$ & 0,799 & 0,240 & 0,782 & 0,233 & 0,765 & 0,221 \\
\hline
\end{tabular}


Observou-se que, para $50^{\circ} \mathrm{C}$, a umidade de equilíbrio do farelo variou de 0,034 a $0,240 \mathrm{~kg} \mathrm{H}_{2} \mathrm{O} / \mathrm{kg}$ sól. seco, em uma faixa de umidade relativa entre 11,1 e $79,9 \%$. Já para $60^{\circ} \mathrm{C}$, a umidade de equilíbrio do farelo variou de 0,029 a $0,233 \mathrm{~kg} \mathrm{H}_{2} \mathrm{O} / \mathrm{kg}$ sól. seco e em uma faixa de umidade relativa entre 11 e $78,2 \%$ e, para $70^{\circ} \mathrm{C}$, a umidade de equilíbrio do farelo variou de 0,023 a $0,221 \mathrm{~kg}$ $\mathrm{H}_{2} \mathrm{O} / \mathrm{kg}$ sól. seco e em uma faixa de umidade relativa entre 10,9 a $76,5 \%$.

Com isso, verificou-se que à medida que a temperatura do ar de secagem aumenta, a umidade de equilíbrio do farelo sofre uma pequena variação para uma determinada umidade relativa do ar, sendo que este comportamento foi observado por GEANKOPLIS [3] e BARROZO [1] em estudos de umidade de equilíbrio para cereais.

Na Tabela 3, são apresentados os resultados dos parâmetros obtidos para cada uma das equações quando ajustadas aos valores experimentais nas condições de 50, 60 e $70^{\circ} \mathrm{C}$. A avaliação do melhor modelo de ajuste se dá pelo maior coeficiente de correlação, $\mathrm{R}^{2}$, e o menor somatório dos desvios quadráticos.

TABELA 3 - Resultados do ajuste para os dados experimentais a 50,60 e $70^{\circ} \mathrm{C}$

\begin{tabular}{lccccc}
\hline \multirow{2}{*}{ Equação } & \multicolumn{3}{c}{ Parâmetros } & \multicolumn{3}{c}{ Coeficientes } \\
\cline { 2 - 6 } & $\boldsymbol{A}_{\mathbf{1}}$ & $\boldsymbol{A}_{\mathbf{2}}$ & $\boldsymbol{A}_{3}$ & $\boldsymbol{R}^{2}$ & $\boldsymbol{\varphi}$ \\
\hline LUIKOV & 0,960 & 0,041 & -- & 0,97 & 0,006 \\
HALSEU & 9,669 & 0,857 & -- & 0,97 & 0,006 \\
HENDERSON/ & 0,076 & $-15,793$ & 1,172 & 0,95 & 0,009 \\
THOMPSON & & & & &
\end{tabular}

A análise da qualidade de ajuste das isotermas de equilíbrio do farelo de soja apresentados na Tabela 3 indica que as equações de HALSEY e de LUIKOV se destacam em relação ao modelo de HENDERSON-THOMPSON, por apresentarem melhor coeficiente de correlação, $R^{2}$. Ou seja, para as equações de HALSEY e LUIKOV os $R^{2}$ obtidos foram de 0,97 , enquanto que para HENDERSON-THOMPSON, foi de 0,95. Assim, para diferenciar os modelos de HALSEY e LUIKOV, calculou-se o somatório dos desvios quadráticos, $\phi$, de cada um e o resultado foi 0,006 para ambos, indicando que os dois modelos são adequados para a representação dos dados de umidade de equilíbrio.

Na Figura 5, são apresentados os dados experimentais da desidratação do farelo de soja classificados de acordo com a temperatura.

A análise do comportamento dos pontos experimentais apresentados na Figura 5 indica que a temperatura exerce pouca influência sobre a umidade de equilíbrio para o farelo de soja na faixa de condições experimentais explorada.

As Figuras 3, 4 e 5 foram construídas com o objetivo de apresentar as isotermas de equilíbrio, obtidas a partir das equações de HENDERSON-THOMPSON, HALSEY, LUIKOV, comparando-as com os dados experimentais nas temperaturas de 50,60 e $70^{\circ} \mathrm{C}$ de forma isolada.
Para tornar o estudo mais completo, somaram-se aos valores experimentais os dados apresentados por PIXTON e WARBURTON [9] para 15, 25 e $35^{\circ} \mathrm{C}$, os quais são apresentados na Tabela 4 e conjuntamente na Figura 6.

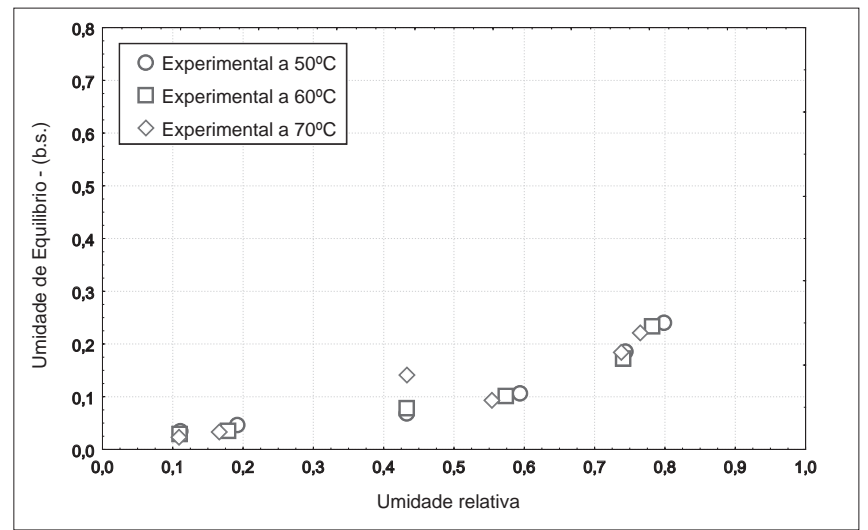

FIGURA 2 - Curvas de equilíbrio ajustadas para o farelo de soja a 50,60 e $70^{\circ} \mathrm{C}$

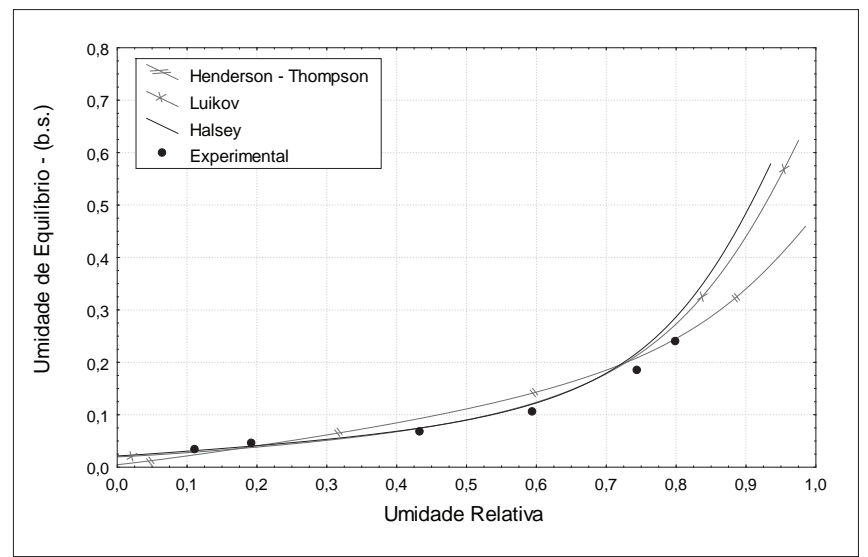

FIGURA 3 - Curvas de equilíbrio ajustadas para o farelo de soja a $50^{\circ} \mathrm{C}$

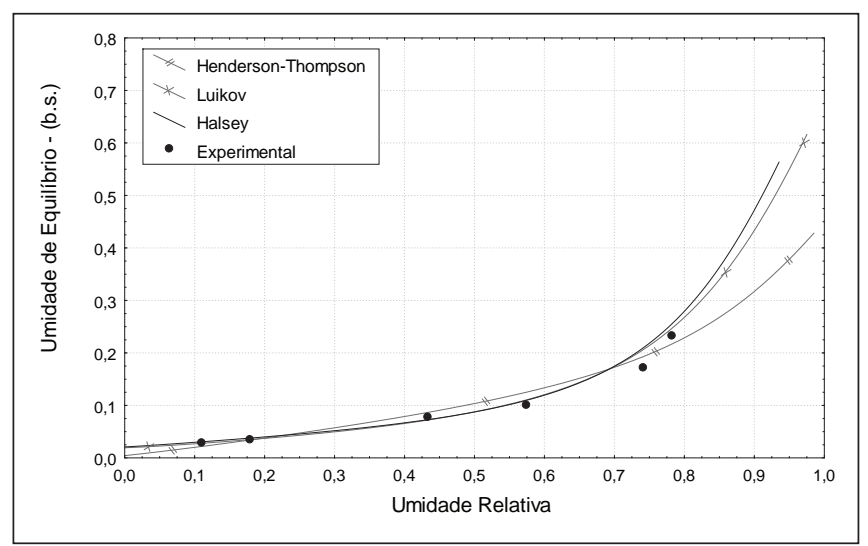

FIGURA 4 - Curvas de equilíbrio ajustadas para o farelo de soja a $60^{\circ} \mathrm{C}$ 


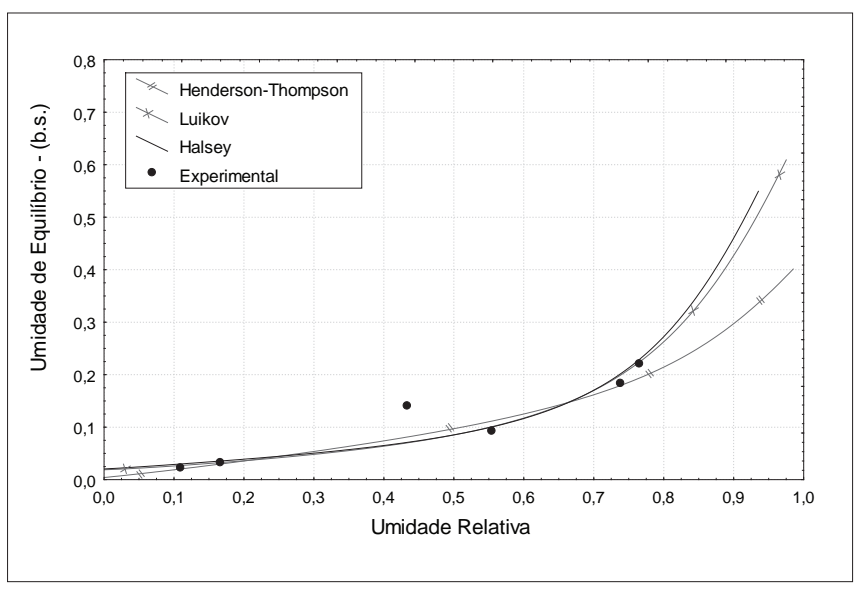

FIGURA 5 - Curvas de equilíbrio ajustadas para o farelo de soja a $70^{\circ} \mathrm{C}$

TABELA 4 - Dados de umidade de equilíbrio para o farelo de soja a 15,25 e $35^{\circ} \mathrm{C}$

\begin{tabular}{cccccc}
\hline \multicolumn{2}{c}{$\mathbf{1 5}^{\circ} \mathbf{C}$} & \multicolumn{2}{c}{$25^{\circ} \mathbf{C}$} & \multicolumn{2}{c}{$35^{\circ} \mathbf{C}$} \\
\hline UR & $\boldsymbol{X}_{\boldsymbol{e}}$ & UR & $\boldsymbol{X}_{\boldsymbol{e}}$ & UR & $\boldsymbol{X}_{\boldsymbol{e}}$ \\
\hline 0,543 & 0,935 & 0,543 & 0,959 & 0,543 & 0,939 \\
0,339 & 0,880 & 0,339 & 0,889 & 0,339 & 0,902 \\
0,222 & 0,794 & 0,222 & 0,792 & 0,222 & 0,786 \\
0,085 & 0,372 & 0,085 & 0,409 & 0,085 & 0,447 \\
0,063 & 0,183 & 0,063 & 0,200 & 0,063 & 0,224 \\
\hline
\end{tabular}

Fonte: Pixton e Warburton (1975)

A Tabela 5 apresenta os parâmetros obtidos para cada uma das equações ajustadas conjuntamente aos dados deste trabalho e aos dados de PIXTON e WARBURTON [9].

TABELA 5 - Resultados do ajuste para $15,25,35,50,60$ e $70^{\circ} \mathrm{C}$

\begin{tabular}{lccccc}
\hline \multirow{2}{*}{ Equação } & \multicolumn{3}{c}{ Parâmetros } & \multicolumn{3}{c}{ Coeficientes } \\
\cline { 2 - 6 } & $\boldsymbol{A}_{\boldsymbol{1}}$ & $\boldsymbol{A}_{\mathbf{2}}$ & $\boldsymbol{A}_{3}$ & $\boldsymbol{R}^{2}$ & $\boldsymbol{\varphi}$ \\
\hline LUIKOV & 0,834 & 0,036 & -- & 0,99 & 0,019 \\
HALSEY & 2,982 & 0,614 & -- & 0,98 & 0,028 \\
HENDERSON/ & 0,023 & $-1,476$ & 0,917 & 0,98 & 0,029 \\
THOMPSON & & & & &
\end{tabular}

Os resultados da Tabela 5 mostram que o melhor modelo matemático é o de LUIKOV, pois apresenta o maior coeficiente de correlação de 0,99 e menor somatório dos desvios quadráticos de 0,019.

A Figura 6 tem a finalidade de mostrar graficamente o grau de ajuste das equações de HALSEY, LUIKOV, HENDERSON-THOMPSON para os todos dados da Tabela 5.

Conforme previamente comentado e, apresentado na Figura 2, a temperatura não exerce influência significativa sobre os dados experimentais de umidade de equilíbrio do farelo de soja na faixa de 50 a $70^{\circ} \mathrm{C}$. Esta observação foi confirmada acrescentando os dados de PIXTON E WARBURTON [9] obtidos na faixa de 15 a $35^{\circ} \mathrm{C}$ e foram apresentados na Figura 6. Desta forma, testaram-se as correlações tradicionais da literatura sem a variável tempe-

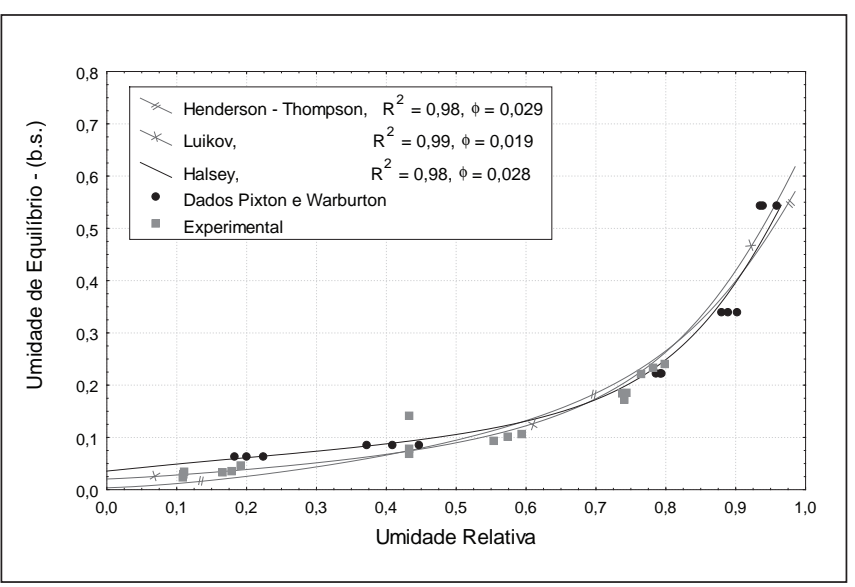

FIGURA 6 - Isotermas de equilíbrio ajustadas para o farelo a 15, $25,35,50,60$ e $70^{\circ} \mathrm{C}$

ratura, conforme a Tabela 6 . Adicionalmente, propôs-se um modelo alternativo que relaciona a umidade de equilíbrio com a umidade relativa de forma exponencial.

TABELA 6 - Modelos de ajuste modificados

Correlação de LUIKOV modificado

$$
X e=\frac{A_{1}}{\left(1+A_{2} \ln \left(\frac{1}{U R}\right)\right)}
$$

Correlação de HALSEY modificado

$$
X e=A_{1}\left(\operatorname{In}\left(\frac{1}{U R}\right)\right)^{-A_{2}}
$$

Correlação de HENDERSON-THOMPSON modificado

$$
X e=\left(\frac{-\ln (1-U R)}{A_{1}}\right)^{\left(\frac{1}{A_{2}}\right)}
$$

Correlação exponencial

$$
X e=A_{1} \exp \left(A_{2} U R\right)
$$

A Tabela 7 apresenta os parâmetros obtidos para cada uma das equações modificadas, ajustadas conjuntamente aos dados deste trabalho e aos dados de PIXTON e WARBURTON [9].

Tabela 7 - Resultados de ajuste para as equações modificadas.

\begin{tabular}{lcccc}
\hline \multirow{2}{*}{ Equação } & \multicolumn{2}{c}{ Parâmetros } & \multicolumn{2}{c}{ Coeficientes } \\
\cline { 2 - 5 } & $\boldsymbol{A}_{\boldsymbol{1}}$ & $\boldsymbol{A}_{\boldsymbol{2}}$ & $\boldsymbol{R}^{\mathbf{2}}$ & $\boldsymbol{\varphi}$ \\
\hline LUIKOV & 0,880 & 12,184 & 0,99 & 0,017 \\
HALSEY & 0,086 & 0,632 & 0,98 & 0,028 \\
HENDERSON-THOMPSON & 5,090 & 0,826 & 0,98 & 0,029 \\
Exponencial & 0,025 & 3,011 & 0,95 & 0,070 \\
\hline
\end{tabular}

Ao comparar as Tabelas 5 e 7, pode-se averiguar que o modelo de LUIKOV continua a ser o mais adequado de acordo com os resultados estatísticos. A diferença pode ser constatada apenas nos somatórios dos desvios quadráticos na terceira casa decimal, comprovando a interferência não significativa da temperatura na estimativa da umidade de equilíbrio do farelo de soja, o que também pode ser 
comprovado pelas Figuras 6 e 7. Apesar de ser proposto um modelo exponencial, ele não mereceu discussão por apresentar resultados insatisfatórios.

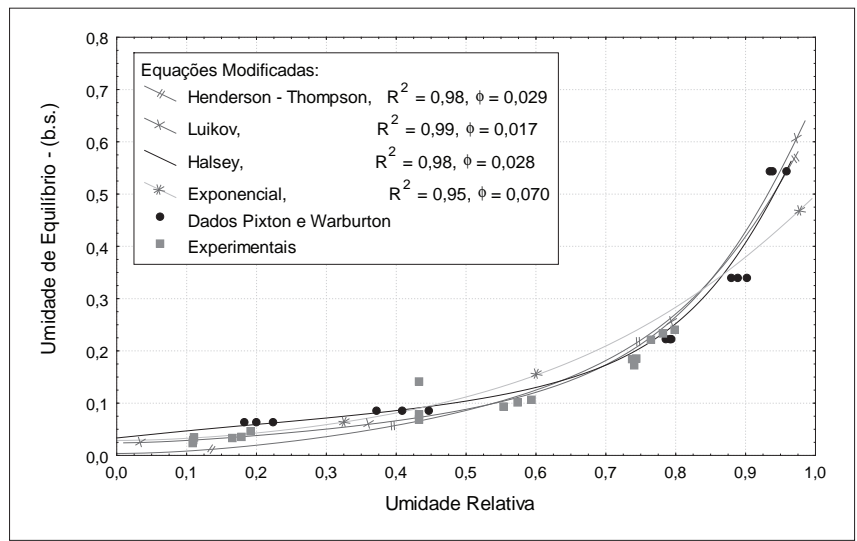

FIGURA 7 - Isotermas de equilíbrio ajustadas e modificadas para o farelo de soja

É interessante notar que, para uma grande faixa de umidade relativa do ar (0 a 60\%), a umidade de equilíbrio do farelo de soja varia muito pouco e permanece em níveis inferiores a $0,15 \mathrm{~kg} \mathrm{H}_{2} \mathrm{O} / \mathrm{kg}$ sól. seco, representando uma faixa operacional atraente para processos industriais de secagem do farelo de soja. Este comportamento também é apresentado por GEANKOPLIS [3] para produtos alimentícios.

Os dados experimentais de 50,60 e $70^{\circ} \mathrm{C}$ também foram utilizados para o ajuste das equações modificadas, cujos parâmetros, coeficientes de correlação e somatório dos desvios médios são mostrados na Tabela 8.

TABELA 8 - Resultados de ajuste para 50, 60 e $70^{\circ} \mathrm{C}$ para os modelos ajustados modificados e exponencial proposto

\begin{tabular}{lcccc}
\hline \multirow{2}{*}{ Equação } & \multicolumn{2}{c}{ Parâmetros } & \multicolumn{2}{c}{ Coeficientes } \\
& $\boldsymbol{A}_{\boldsymbol{1}}$ & $\boldsymbol{A}_{\mathbf{2}}$ & $\boldsymbol{R}^{\mathbf{2}}$ & $\boldsymbol{\varphi}$ \\
\hline LUIKOV & 1,048 & 15,093 & 0,97 & 0,005 \\
HALSEY & 0,066 & 0,867 & 0,97 & 0,005 \\
HENDERSON-THOMPSON & 7,689 & 1,051 & 0,96 & 0,007 \\
Exponencial & 0,021 & 2,970 & 0,97 & 0,006 \\
\hline
\end{tabular}

Na Tabela 8, observou-se que os modelos de HALSEY e LUIKOV modificados continuam os mais adequados para estimar a umidade de equilíbrio do farelo de soja.

As isotermas de equilíbrio do farelo de soja, de acordo com os parâmetros apresentados na Tabela 8, são mostradas na Figura 8.

Ao comparar os modelos originais e modificados para os dados nas condições de 15, 25, 35, 50, 60 e $70^{\circ} \mathrm{C}$, averiguou-se que LUIKOV apresentou os melhores coeficientes de ajuste e para tanto, ambos podem ser observados na Figura 9.

Pela Figura 9, nota-se que a diferença entre os resultados previstos para os modelos aparece quando a umidade relativa está na faixa de 0,8 a 1 .

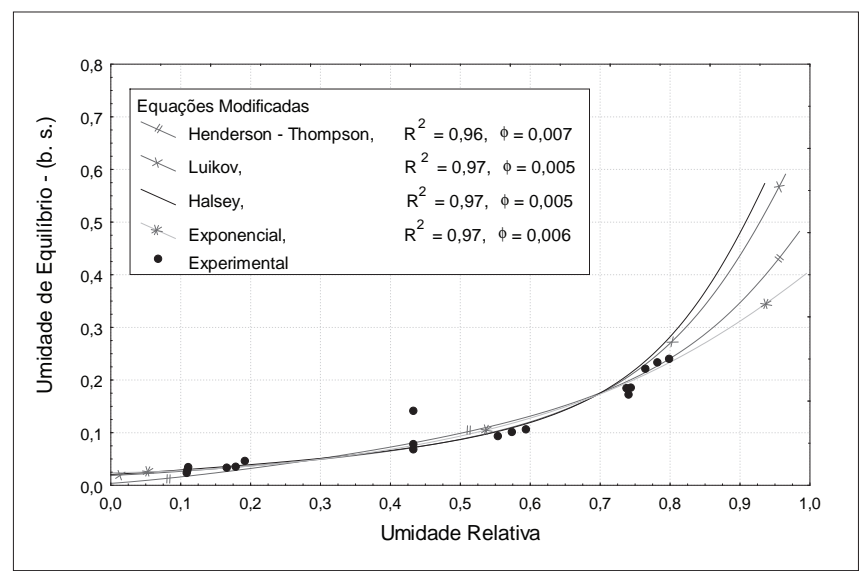

FIGURA 8 - Curvas de equilíbrio utilizando as correlações ajustadas e modificadas com dados experimentais para o farelo de soja

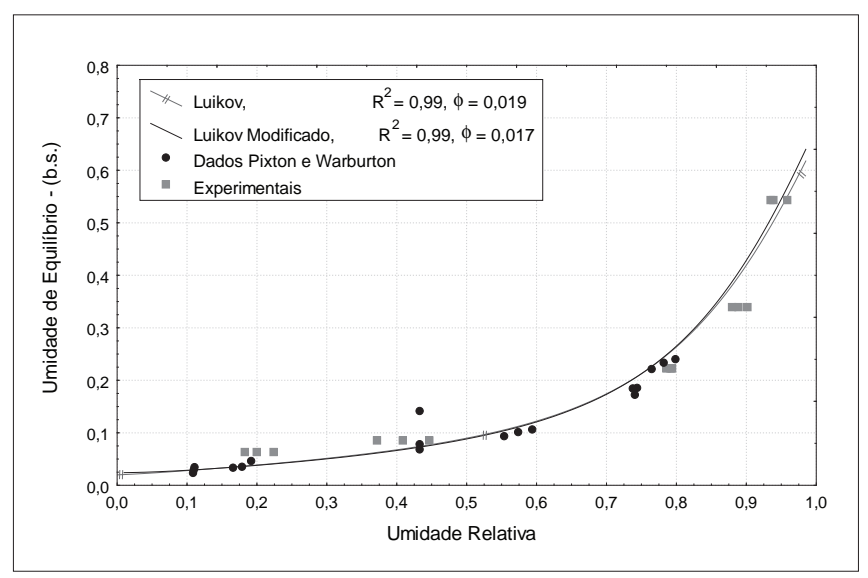

FIGURA 9 - Curvas comparativas de equilíbrio utilizando a correlação ajustada de LUIKOV

\section{4 - CONCLUSÕES}

Os dados experimentais para o farelo de soja indicam que as equações de HALSEY e de LUIKOV são as mais adequadas para predizer a umidade de equilíbrio do sólido.

Ao comparar as isotermas de equilíbrio para as diferentes condições de temperatura, verificou-se que esta exerce pouca influência sobre a umidade de equilíbrio do farelo de soja, sendo comprovada gráfica e estatisticamente pelos modelos na forma modificada ou original.

Pode-se observar que, de acordo com isotermas construídas, para uma grande faixa de umidade relativa do ar (0 a 60\%), a umidade de equilíbrio do farelo de soja varia muito pouco e permanece em níveis inferiores a $0,15 \mathrm{~kg}$ $\mathrm{H}_{2} \mathrm{O} / \mathrm{kg}$ sól. seco.

\section{5 - NOMENCLATURA}

$A_{1}, A_{2}, A_{3}$ - Parâmetros de ajuste

$R^{2}$ - Coeficiente de correlação

$T$ - Temperatura absoluta do ar (K) 
UR - Umidade relativa do ar (\%/100)

$X_{e}$ - Umidade de equilíbrio do farelo $\mathrm{kg} \mathrm{H}_{2} \mathrm{O} / \mathrm{kg}$ sól. seco

$\Phi$ - Somatório dos desvios quadráticos

\section{6 - REFERÊNCIAS BIBLIOGRÁFICAS}

[1] BARROZO, M.A.S.; OLIVEIRA, D.T., SANCINETI, G.P., RODRIGUES, M.V. A study of the desorption isotherms of lentils. Brazilian Journal of Chemical Engineering, v. 17, p. 105-109, 2000.

[2] BROOKER, D.B.; BAKKER-ARKEMA, F.W.; HALL, C.W. Drying and storage of grains and oilseeds. The AVI Publishing Company, New York, p. 450, 1992.

[3] GEANKOPLIS, C. Transport processes and unit operations. USA: Prentice Hall, 1993.

[4] GREENSPAN, L. Humidity fixed - points of binary satured aqueous solutions. Journal Res. Natl. Bureau of Standards, 81(A), p. 89-93, 1977.

[5] JONGENEELEN, H.P.J. Energy conservation in solvent extration plants, JAOCS Journal of the American Oil Chemists Society, v .53, p. 291, June, 1976.

[6] MUJUNDAR, A.S. Handbook of industrial drying. New York: Marcel Dekker, Inc., 1987.
[7] PARAÍSO, P.R. Modelagem e análise do processo de obtenção do óleo de soja. Campinas, 2001, 200 p. Tese de doutorado, Faculdade de Engenharia Química, Universidade de Campinas (Unicamp), 2001.

[8] PERRY, R.H.; CHILTON, C.H. Manual de Engenharia Química, 5a ed. Rio de Janeiro, Guanabara Dois S. A, 1980.

[9] PIXTON, S.W.; WARBURTON. The moisture content/ equilibrium relative humidity relationship of soya meal. Journal of Stored Products Res. Report, n. 1.109, USDA, 1975.

[10] SARTORI, A.E.R. Modelagem, simulação e controle de um secador rotativo industrial. Maringá, 2001, 92 p. Dissertação (mestrado em Desenvolvimento de Processos), Faculdade de Engenharia Química, Universidade Estadual de Maringá (UEM), agosto, 2001.

[11] SOUSA, L.H.C.D.; PERRI, V.R.S.; PEREIRA, N.C.; MOTTA LIMA, O.C.; MENDES, E.S. Isotermas de equilíbrio para tecidos de algodão - adsorção e dessorção. In: XV Congresso Brasileiro de Engenharia Química, 2004, Curitiba, Paraná.

[12] TEIXEIRA, C.G. A soja na alimentação humana. Disponível em: http://www.vegetarianismo.com.br/artigos/sojal.html. Acesso em 18 abr. 2005. 